\begin{tabular}{|c|l|}
\hline Title & Plasma leptin concentration in dogs: effects of body condition score, age, gender and breeds \\
\hline Author(s) & Ishioka, K.; Hosoya, K.; Kitagawa, H.; Shibata, H.; Honjoh, T.; Kimura, K.; Saito, M. \\
\hline Citation & $\begin{array}{l}\text { Research in Veterinary Science, 82(1), 11-15 } \\
\text { https://doi.org/10.1016/.rvsc.2006.06.002 }\end{array}$ \\
\hline Issue Date & $2007-02$ \\
\hline Doc URL & http://hdl.handle.net/2115/18914 \\
\hline Type & article (author version) \\
\hline File Information & RV S82-1.pdf \\
\hline
\end{tabular}

Instructions for use 


\title{
Plasma leptin concentration in dogs: effects of body condition score, age, gender and breeds
}

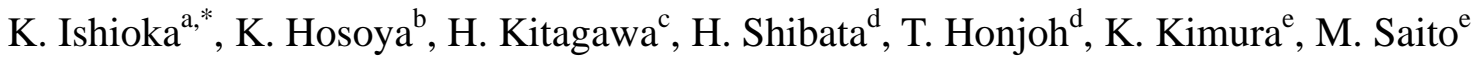

${ }^{a}$ Department of Veterinary Nursing, School of Veterinary Nursing \& Technology, Faculty of Veterinary Science, Nippon Veterinary and Life Science University, Tokyo 180-8602, Japan

${ }^{\mathrm{b}}$ Laboratory of Surgery, Department of Veterinary Clinical Sciences, Graduate School of

Veterinary Medicine, Hokkaido University, Sapporo 060-0818, Japan

${ }^{c}$ Laboratory of Veterinary Internal Medicine, Faculty of Applied Biological Sciences, Gifu

University, 1-1, Yanagido, Gifu 501-1193, Japan

dMorinaga Institute of Biological Science, Inc.; 1-16 Sachiura 2-chome, Kanazawa-ku, Yokohama 236-0003, Japan

${ }^{\mathrm{e}}$ Laboratory of Biochemistry, Department of Biomedical Sciences, Graduate School of Veterinary Medicine, Hokkaido University, Sapporo 060-0818, Japan

${ }^{*}$ Corresponding author

Present address:

Department of Veterinary Nursing, School of Veterinary Nursing \& Technology,

Faculty of Veterinary Science, Nippon Veterinary and Life Science University

1-7-1, Kyonan-cho, Musashino-shi, Tokyo 180-8602, Japan 
Tel: +81-422-31-4151

Fax: +81-422-33-2094

E-mail: katsumi@nvau.ac.jp

Running head: Leptin in obese dogs.

\begin{abstract}
Leptin is a cytokine produced by adipocytes, and plays a key role in the regulation of energy balance. In the present study, we measured plasma leptin concentrations of 166 normal and obese dogs visiting veterinary practices, and clarified the influence of age, gender and breed on plasma leptin levels in dogs. Leptin levels were higher in the dogs with higher body condition scores. There was no noticeable influence of age, gender and breed, but those in optimal puppies and obese Miniature Dachshund tended to be lower than those in corresponding groups. We conclude that plasma leptin is a reliable marker of adiposity in dogs regardless of age, gender and breed variations, and thereby useful as a blood biochemistry test for health examinations and treatment of obesity.
\end{abstract}


Key words: adipocytokine; diabetes; dog; leptin; obesity

\section{Introduction}

Obesity is the most common nutritional disorder encountered in small animal medicine, and is associated with various other diseases, such as diabetes mellitus, pancreatitis, cardiovascular diseases and articular problems (Lund et al., 1999). Quantitative estimation of adiposity is essential for diagnosing obesity and for evaluation of preventive and therapeutic challenges in human medicine. Although there are various methods for diagnosis of obesity in human medicine, most of them are not successfully applicable to small animal medicine. For example, body mass index (BMI) is a simple and reliable indicator of obesity in humans, but it is not suitable in the dog and cat because of wide variations of their body dimensions. The body condition score (BCS) and relative body weight (RBW) are widely used to evaluate nutritional condition of the dog and cat, but they are based on subjective estimation (Laflamme, 1997).

Leptin, the product of the $o b$ gene, is a representative adipocytokine, which is synthesized and secreted primarily by adipocytes (Zhang et al., 1994), and it is a key molecule for the regulation of whole body energy balance. In humans and rodents, blood leptin concentration is known to positively correlate with body fat content (Maffei et al., 1995). Since plasma leptin 
level is closely related to metabolic condition, it can be a strong tool for the study of obesity and related disorders. Previously, we cloned canine leptin gene (Iwase et al., 2000b), produced recombinant protein, and established a sandwich ELISA specific to canine leptin (Iwase et al., 2000a). Using this ELISA method, we demonstrated a highly positive correlation between plasma leptin concentration and body fat content estimated by a deuterium oxide dilution method in experimentally induced obese beagles (Sagawa et al., 2002, Ishioka et al., 2002b). We also found, in preliminary studies, higher plasma leptin concentrations in dogs with higher BCSs visiting veterinary practices (Ishioka et al., 2002b), but the sample size was too small to analyse the effects of various factors other than BCSs statistically. In the present study, we collected plasma samples from 166 dogs visiting 8 veterinary practices and performed a retrospective study focusing on the relationship of the plasma leptin concentration to BCS, age, gender and breed.

\section{Materials and Methods}

\subsection{Subjects and sample collection}

Plasma samples were collected during a 5-year period from 1999 to 2003 from eight veterinary practices in Japan consisting of two veterinary teaching hospitals (Hokkaido University and Gifu University) and 6 private general practices. Detailed information, such as name, age, gender (male, female, castrated or spayed), breed, feeding status (fed or fasted before 
sampling) and clinical history were recorded for each dog. The assessment of the nutritional condition was based on a five-scale BCS. Since we have previously found that plasma leptin concentration is increased $2-10$ hours (hr) after food intake (Ishioka et al., 2005), administration of insulin (Ishioka et al., 2005) and glucocorticoids (Ishioka et al., 2002a), in the present study, we carefully assessed the individual records, and only used blood samples collected from 166 dogs that had been fasted for at least $10 \mathrm{hr}$ and not treated with insulin and/or glucocorticoids. Most of the animals presented to the hospitals for castration, ovariohysterectomy, dental treatment and health examinations.

\subsection{Leptin assay}

The plasma leptin concentration was measured by the previously reported method of sandwich ELISA using an anti-canine leptin antibody (Iwase et al., 2000a) with minor modifications. Briefly, each well of a 96-well microplate was coated with $0.2 \mu \mathrm{g}$ of the purified rabbit anti-canine leptin antibody and incubated for $2 \mathrm{hr}$ at room temperature. After washing the wells twice with TBS-T (10 mM Tris-HCl, pH 7.4/150 mM NaCl/0.05\% Tween 20), $300 \mu \mathrm{L}$ of TBS-T-BSA (TBS-T/0.1\% bovine serum albumin) was added to each well for blocking. After a $2 \mathrm{hr}$ incubation, the wells were then washed twice with TBS-T, and incubated overnight at $4^{\circ} \mathrm{C}$ with $80 \mu \mathrm{L}$ of $30 \%$ normal rabbit serum and either $20 \mu \mathrm{L}$ of plasma or recombinant canine leptin (0.5 - $32 \mathrm{ng} / \mathrm{mL}$ ). The wells were washed 5 times with TBS-T, and incubated with $100 \mu \mathrm{L}$ of horseradish peroxidase-conjugated anti-canine leptin antibody $(0.2 \mu \mathrm{g} / \mathrm{mL})$ for $4 \mathrm{hr}$ at $4^{\circ} \mathrm{C}$. 
After washing 7 times, the wells were incubated with $100 \mu \mathrm{L}$ of a peroxidase substrate solution in the dark for $30 \mathrm{~min}$ at room temperature. After stopping the reaction by adding $50 \mu \mathrm{L}$ of $1 \mathrm{~N}$ sulfuric acid, the absorbance of each well was measured at $450 \mathrm{~nm}$.

\subsection{Data analyses}

All leptin data were recorded and classified based on their profiles, such as age, gender, breed and BCS. Values were expressed as mean \pm standard error of the mean. Statistical analyses were performed by Spearman's rank correlation test for comparison of the groups with different BCSs. Analysis of variance (ANOVA) with Scheffe's test as a post hoc was also used for comparison of the groups with different profiles, such as gender and breed. $\quad \mathrm{P}<0.05$ is considered significant. (Statview 5.0, SAS Institute Inc, Cary, NC, USA)

\section{Results}

\subsection{Population of subjects}

The general profiles and populations of 166 dogs used in the present study were as follows: When the dogs were divided based on their BCS, 45 were optimal (BCS 3), 46 overweight (BCS 4) and 75 obese (BCS 5). Their ages ranged between 6 months and 17 years old, with a median of 8.8 years old. Fourty-eight were intact males, 12 castrated, 57 intact female and 44 spayed 
(the age of 5 dogs and the gender of 15 dogs were unknown). The breeds included 31 Golden Retriever, 10 Labrador Retriever, 26 Miniature Dachshunds, 15 Shetland Sheepdog, 13 Shih Tzu and 34 mixed breeds. The other 30 dogs consisted of Beagle, Bichon Frise, Chihuahua, Cocker Spaniel, Maltese, Miniature Schnauzer, Pekingese, Pomeranian, Poodle, Shiba Inu, Siberian Husky, Tosa Inu, Yorkshire Terrier, and Welsh Corgi, but the number of these individual breeds was less than 10 each.

\subsection{Effects of BCS}

Table 1 shows plasma leptin concentrations for each BCS in all dogs. Plasma leptin concentrations were considerably different among individual dogs, ranging from undetectable (less than 0.5 ) to $33.4 \mathrm{ng} / \mathrm{mL}$. Mean leptin concentrations were $3.0 \pm 0.2 \mathrm{ng} / \mathrm{mL}$ in BCS 3, $8.6 \pm$ $0.7 \mathrm{ng} / \mathrm{mL}$ in BCS 4 and $12.8 \pm 0.8 \mathrm{ng} / \mathrm{mL}$ in BCS 5. Plasma leptin concentrations were significantly higher in dogs with higher BCSs $(\rho=0.639, \mathrm{p}<0.0001)$.

\subsection{Effects of age and gender}

Table 2 shows the correlation between the plasma leptin concentration and age for each BCS in 151 dogs (the age of 15 subjects was unknown). There was a weak but positive correlation between plasma leptin concentration and age in the 40 dogs with BCS $3(\rho=0.345$, $\mathrm{p}=0.031$ ). This group included 6 puppies younger than 1 year old, which showed rather lower leptin concentration $(2.0 \pm 0.2 \mathrm{ng} / \mathrm{mL})$ than the remaining 34 adults $(3.1 \pm 0.3 \mathrm{ng} / \mathrm{mL})$. When 
the data from only the 34 adults with BCS 3 were analyzed, no significant relationship was detected. Similarly, there was no significant effects of age in dogs with BCS $4(p=0.609)$ and 5 $(\mathrm{p}=0.263)$

The effects of gender were also analyzed in 161 dogs (the gender of 5 subjects was unknown). As shown in Table 3, plasma leptin concentrations were not different between male and female dogs regardless of their BCS. In male dogs, there was no significant effect of castration regardless of the BCS. Although spayed dogs of BCS 3 showed elevated plasma leptin concentrations compared to unspayed females ( $4.8 \pm 0.8 \mathrm{ng} / \mathrm{mL}$ vs. $2.6 \pm 0.3 \mathrm{ng} / \mathrm{mL})$, it was not significant and such effects were not found in female dogs with BCS 4 and $5 . \quad$ More importantly, in all genders, plasma leptin showed significantly higher values in dogs with higher BCSs $(\rho=0.512-0.704, p=<0.0001-0.042)$.

\subsection{Plasma leptin in various breeds}

Table 4 shows the relationship between the plasma leptin concentration and BCS in various breeds. Six breeds, Miniature Dachshund (n=26), Shih Tzu (n=13), Mix breed (n=34), Shetland Sheepdog $(n=15)$, Golden Retriever $(n=31)$, and Labrador Retriever $(n=10)$, were selected for the analysis, while the data from other breeds were not analyzed because of rather small sample size

of less than 10 dogs per breed. In dogs with BCS 3, the mean plasma leptin concentrations were equal to or lower than $5 \mathrm{ng} / \mathrm{mL}$ in all the breeds, showing no significant difference among the breeds. Plasma leptin concentrations in dogs with BCS 4 ranged between $7.1 \pm 1.2 \mathrm{ng} / \mathrm{mL}$ and 
$15.5 \pm 2.6 \mathrm{ng} / \mathrm{mL}$, and those in dogs with BCS 5 were between $8.1 \pm 1.2 \mathrm{ng} / \mathrm{mL}$ and $21.0 \pm 3.1$

ng/mL. In all BCS groups, Shetland Sheepdog showed the highest leptin values while Miniature Dachshund showed the lowest. Plasma leptin at BCS 5 in Miniature Dachshund was significantly lower than that in Shetland Sheepdog $(\mathrm{P}<0.05)$. More importantly, in all breeds, plasma leptin concentrations were significantly higher in dogs with higher BCSs ( $\rho=0.558-0.812$, $\mathrm{p}=0.001-0.037)$.

\section{Discussion}

We have shown that plasma leptin concentrations correlate positively with body fat content in experimentally induced obese beagles (Sagawa et al., 2002, Ishioka et al., 2002b), suggesting that plasma leptin is a good index of adiposity in the dog, as has been well-establised in humans and rodents. In our previous studies using beagles, the subjects were chosen for homogeneity of age, gender, breed, and housing conditions such as room temperature, lighting conditions, and feeding time. In small animal practices, however, the patients and their conditions are considerably heterogeneous. In preliminary studies using 19 overweight and/or obese dogs visiting veterinary practices, we also found elevated plasma leptin concentrations compared with non-obese optimal weight dogs (Ishioka et al., 2002b). Consistent with our preliminary results, the present large-scale retrospective study using 166 clinical cases clearly showed higher plasma 
leptin concentrations in subjects with higher BCSs; that is, the mean concentration was $3.0 \mathrm{ng} / \mathrm{mL}$ in BCS 3, and increased to 8.6 and 12.8 in BCS 4 and 5, respectively. Since BCS is widely accepted as a semi-quantitative index of adiposity, and BCS 3, 4 and 5 correspond to optimal, overweight and obese, respectively, plasma leptin is thus confirmed to be a quantitative marker of obesity in the dog.

Based on the relatively large number of subjects, we analyzed the influences of age, gender and breed on the BCS-related increase in plasma leptin. The analysis of the relationship between the plasma leptin concentration and age at each BCS showed a weak but significant positive correlation between the two parameters in 40 dogs with BCS 3, but not those with BCS 4 and 5. The BCS 3 group contained 6 puppies younger than 1 year old, which showed lower leptin concentrations than the remaining 34 adults, probably reflecting less body fat contents in puppies than that in the adult dog. In fact, re-analysis of the data after censoring the puppies revealed no significant effect of age, in the same way as in the groups of BCS 4 and 5 which did not include puppies. Thus, there is no effect of age on plasma leptin concentration and its relation to adiposity over one year of age (generally, obesity is a disorder of adult subjects).

The effects of gender physiology were analyzed by comparing the leptin data obtained from male, female or between neutered and intact dogs. There was no significant difference between male and females, and also between neutered (castrated or spayed) and normal dogs, regardless of whether they were obese or of optimal body condition. These findings seem consistent with our previous results using female beagles in which plasma leptin concentrations 
did not change after ovariohysterectomy (Sagawa et al., 2002). In humans and mice, it has been reported that plasma leptin concentrations are higher in females than males, depending on leptin secreted from the placenta (Masuzaki et al., 1997). Although our results did not confirm such a gender difference, we have not yet measured leptin levels in pregnant dogs. Further study is required to clarify this point. In addition, stomach (Bado et al., 2005) and mammary gland (Smith-Kirwin et al., 1998) are also known to produce significant amounts of leptin, but these have not been studied as yet in the dog.

Most interesting is that plasma leptin concentrations in the subjects with BCS 3 were within a rather low and limited range in various breeds, despite their largely different body sizes, and increased 3-5 fold with increasing BCS in most breeds tested. One exception was the Miniature Dachshund, in which plasma leptin concentration was lower than $10 \mathrm{ng} / \mathrm{mL}$ even in the subjects with BCS 5. This finding may not be due to their relatively small body size, because plasma leptin in Shih Tzu with BCS 5, which is also a toy-breed, was elevated similarly to other breeds with larger body sizes. We could not detect a significant difference between Miniature Dachshund and other breeds, however, this may in part be due to the small sample size in the individual breed groups. However, plasma leptin concentrations were significantly higher in dogs with higher BCSs in all breeds. The present results strongly suggest that plasma leptin is a good quantitative marker of adiposity, regardless of wide variations of body size among breeds, although the possibility of some innate breed-specific effect cannot be excluded at present. In conclusion, plasma leptin is an available and quantitative index of adiposity in dogs 
regardless of their age, gender and breed variations. In companion animal practices, leptin levels could be monitored in obesity-control programs to ensure fat loss instead of muscle loss, and therefore useful as a blood biochemistry test. In addition, leptin could also be a helpful parameter for scientists studying obesity in dogs, for example, it can be an index to evaluate the effect of newly developed anti-obese drugs. Collectively, leptin is a strong tool for both study of obesity and health examinations in dogs.

\section{Acknowledgements}

This work was supported in part by a grant from Japan Forum on Small Animal Clinical Nutrition (JFSACN). The authors thank Dr. Yoshinori Shimamoto and Dr. Kazuko Inazawa for sampling assistance.

\section{References}

Bado, A., Levasseur, S., Attoub, S., Kermorgant, S., Laigneau, J. P., Bortoluzzi, M. N., Moizo, L., Lehy, T., Guerre-Millo, M., Le Marchand-Brustel, Y., Levin, M. J., 2005. The stomach is a source of leptin. Nature 394, 790-793. 
Ishioka, K., Soliman, M. M., Honjoh, T., Shibata, H., Kimura, K., Saito, M., 2002a.

Dexamethasone increases serum leptin concentration in dogs. The Veterinary Journal 164, 295-297.

Ishioka, K., Soliman, M. M., Sagawa, M., Nakadomo, F., Shibata, H., Honjoh, T., Hashimoto, A., KitamuraI, H., Kimura, K., Saito, M., 2002b. Experimental and clinical studies on plasma leptin in obese dogs. Journal of Veterinary Medical Science 64, 349-353.

Ishioka, K., Hatai, H., Komabayashi, K., Soliman, M. M., Shibata, H., Honjoh, T., Kimura, K., Saito, M., 2005. Diurnal variations of serum leptin in dogs: effects of fasting and re-feeding. The Veterinary Journal 169, 85-90.

Iwase, M., Kimura, K, Komagome, R., Sasaki, N., Ishioka, K., Honjoh, T., Saito, M., 2000a. Sandwich enzyme-linked immunosorbent assay of canine leptin. Journal of Veterinary Medical Science 62, 207-209.

Iwase, M., Kimura, K., Sasaki, N., Komagome, R., Ishioka, K., Morimatsu, M., Murakami, T., Saito, M., 2000b. Canine leptin: cDNA cloning, expression and activity of recombinant protein. Research in Veterinary Science 68, 109-114. 
Laflamme, D., 1997. Development and validation of a body condition score system for dogs. Canine Practice 22, 10-15.

Lund, E. M., Armstrong, P. J., Kirk, C. A., Kolar, L. M., Klausner, J. S., 1999. Health status and population characteristics of dogs and cats examined at private veterinary practices in the United States. Journal of the American Veterinary Medical Association 214, 1336-1341.

Maffei, M., Halaas, J., Ravussin, E., Pratley, R. E., Lee, G. H., Zhang, Y., Fei, H., Kim, S., Lallone, R., Ranganathan, S., Kern, P. M., Friedman, J. M., 1995. Leptin levels in human and rodent: Measurement of plasma leptin and ob RNA in obese and weight-reduced subjects. Nature Medicine 1, 1155-1161.

Masuzaki, H., Ogawa, Y., Sagawa, N., Hosoda, K., Matsumoto, T., Mise, H., NishimuraI, H., Yoshimasa, Y., Tanaka, I., Mori, T., Nakao, K., 1997. Nonadipose tissue production of leptin: leptin as a novel placenta-derived hormone in human. Nature Medicine 3, 1029-1033.

Sagawa, M. M., Yoneda, S., Nakadomo, F., Honjoh, T., Ishioka, K., Saito, M., 2002. Correlation between plasma leptin concentration and body fat content in dogs. American Journal of Veterinary Research 63, 7-10. 
Smith-Kirwin, S. M., O’Conner, D. M., De Johnston, J, Lancey, E. D., Hassink, S. G., Funanage, V. L., 1998. Leptin expression in human mammary epithelial cells and breast milk. Journal of Clinical Endocrinology and Metabolism 83, 1810-1813.

Zhang, Y., Proenca, R., Maffei, M., Barone, M., Leopold, L., Friedman, J. M., 1994. Positional cloning of the mouse obese gene and its human homologue. Nature 372, 425-431. 


\section{Table 1}

Plasma leptin concentration for each body condition score (BCS).

\begin{tabular}{ll}
\hline BCS & Leptin $(\mathrm{ng} / \mathrm{mL})$ \\
3 & $3.0 \pm 0.4(\mathrm{n}=45)$ \\
4 & $8.6 \pm 0.7(46)$ \\
5 & $12.8 \pm 0.8(75)$ \\
$\rho=0.639(\mathrm{p}<0.0001)$
\end{tabular}

\section{Table 2}

Correlation between the plasma leptin concentration and age for each body condition score (BCS).

\begin{tabular}{lll}
\hline BCS & $\rho$ (leptin vs age) & $\mathrm{p}$ \\
\hline 3 & $0.345(\mathrm{n}=40)$ & 0.031 \\
4 & $0.082(40)$ & 0.609 \\
5 & $0.134(71)$ & 0.263 \\
\hline
\end{tabular}


Table 3

Gender variation of the plasma leptin concentration for each body condition score (BCS).

\begin{tabular}{lllll}
\hline Gender & \multicolumn{4}{c}{ BCS } \\
\cline { 2 - 4 } & 3 & 4 & 5 & \\
\hline Intact male & $3.0 \pm 0.4(\mathrm{n}=19)$ & $8.7 \pm 1.4(14)$ & $12.7 \pm 1.7(15)$ & $\rho=0.704(\mathrm{p}<0.0001)$ \\
Castrated & $1.3 \pm 0.8(2)$ & $12.5 \pm 2.9(5)$ & $16.0 \pm 64.1(5)$ & $\rho=0.614(\mathrm{p}=0.042)$ \\
Intact female & $2.6 \pm 0.3(14)$ & $7.3 \pm 1.0(16)$ & $12.1 \pm 1.4(27)$ & $\rho=0.663(\mathrm{p}<0.0001)$ \\
Spayed & $4.8 \pm 0.8(5)$ & $8.7 \pm 1.1(11)$ & $12.9 \pm 1.4(28)$ & $\rho=0.512(\mathrm{p}=0.0008)$ \\
\hline
\end{tabular}




\section{Table 4}

Relationship between the plasma leptin concentration (ng/mL) and body condition score (BCS) among various breeds $(n=129)$.

\begin{tabular}{lllll}
\hline Breed & \multicolumn{4}{l}{ BCS } \\
\cline { 2 - 4 } & 3 & 4 & 5 \\
\hline Miniature Dachshund & $2.5 \pm 0.4(\mathrm{n}=8)$ & $7.1 \pm 1.2(10)$ & $8.1 \pm 1.2^{\mathrm{a}}(8)$ & $\rho=0.685(\mathrm{p}=0.001)$ \\
Shih Tzu & $2.8 \pm 0.4(6)$ & $8.2 \pm 2.1(3)$ & $14.8 \pm 6.3(4)$ & $\rho=0.812(\mathrm{p}=0.005)$ \\
Mix & $2.6 \pm 1.0(6)$ & $10.1 \pm 2.0(8)$ & $13.0 \pm 1.8(20)$ & $\rho=0.558(\mathrm{p}=0.001)$ \\
Shetland Sheepdog & $5.0 \pm 1.5(3)$ & $15.5 \pm 2.6(2)$ & $21.0 \pm 3.1^{\mathrm{b}}(10)$ & $\rho=0.680(\mathrm{p}=0.011)$ \\
Golden Retriever & $4.4 \pm 1.3(5)$ & $12.5 \pm 1.9(10)$ & $15.4 \pm 1.1(16)$ & $\rho=0.590(\mathrm{p}=0.001)$ \\
Labrador Retriever & $3.1 \pm 0.5(3)$ & $7.9 \pm 2.6(5)$ & $13.4 \pm 1.4(2)$ & $\rho=0.697(\mathrm{p}=0.037)$
\end{tabular}

a,b Values with different letters are significantly different $(\mathrm{p}<0.05)$. 